\title{
Further studies on the constant stiffness method of non-linear analysis of concrete structures
}

\author{
W. DUNCAN \& T. JOHNARRY
}

Dr R. J. Cope and Dr P. V. Rao. University of Liverpool

We agree that the constant stiffness method converges slowly and is expensive to use. However, we disagree that the way to overcome the problem is to neglect static equilibrium. Unless equilibrium conditions are satisfied to within a reasonable tolerance solutions will be subjective. An analyst could produce a range of solutions and have no criteria with which to distinguish the correct one.

39. If attempts to satisfy equilibrium often lead to poor results, as the Authors suggest, then the modelling of material properties should be examined. Both the Authors' and our analyses ${ }^{1}$ set concrete stresses normal to cracks to zero. Although this is true for plain concrete, the presence of tension stiffening in reinforced concrete ensures the carrying of tensile stresses by the concrete teeth in between cracks. Also the effects of aggregate interlock and dowel action provide additional shear stiffness to that suggested by equation (5). When tension stiffening is included in analysis the sudden release of energy illustrated in fig. 11 of reference 1 disappears and results are closer to experimental observations.

40. When the tension stiffening effect is not modelled, analysis predicts an overflexible response. Performing insufficient iterations to give equilibrium results in prediction of an overstiff response. When both features are combined in an analysis the effects tend to cancel out and an apparently accurate model is produced. ${ }^{14}$

41. Can the Authors amplify $\S 10$ ? In a constant stiffness analysis using the initial uncoupled stiffness matrix the median plane is taken as the fixed reference surface for nodal forces and displacements. Movement of the neutral surfaces is achieved by in-plane nodal forces acting on this reference plane. These forces are an essential part of an analysis. When equilibrium is approximately satisfied $N$ in equation (18) is approximately zero, unless in-plane constraints exist, as is the case with edge beams and for slab assemblages.

42. Details of techniques for modelling material properties and of convergence are given in reference 15 .

Paper published: Proc. Instn. Civ. Engrs, Part 2, 1979, 67, Dec., 951-969. 


\section{Dr Duncan and Mr Johnarry}

In the literature a one-step Newton-Raphson correction solution approach has been recorded. ${ }^{16}$ We have relied largely on this; it implies that very close equilibrium state is not demanded at each loading stage. However, the application of the load in smaller increments is a compensating factor.

44. In $\S 39$ the phenomenon of aggregate interlock and dowel action in cracked elements and how this could be used to modify the modelling of material properties was mentioned. This could be done but a new set of parameters requiring further study would have to be introduced.

45. Where in-plane and bending effects are coupled through the constitutive matrices the rate of propagation of cracks would often be extensive initially. Once a good number of the concrete layers have yielded through double cracking the non-linearity is due largely to elasto-plastic microcracking action in the biaxial compressive zone of the failure surface and also in the yielding of the steel. Our method of treating non-linearity in these arrears did not lead to large iterations.

46. For stress-free edges equation (18) should hold in any element of the assemblage at each stage in the solution. In the instance of all the concrete in a slab element yielding, leaving the steel, equation (18) would reduce to

$$
N=\int_{-t / 2}^{t / 2} \sigma \mathrm{d} z=f_{\mathrm{st}} \mathrm{d} s
$$

and this should be the stage of incipient collapse for small displacement analysis. If edge beams are present the out of balance in-plane forces in the slab elements would be transferred into transverse bending of the edge beams and the failure load is now also dependent on the yielding of the stiff edge beams.

47. On page 953 of the Paper, equation (8) should read

$$
\tau_{\text {oct }}+0 \cdot 1714 p-0 \cdot 4143 f_{y}=0
$$

\section{References}

14. COPE R. J. Non-linear analysis of slab assemblages using numerical integration. Proc. Int. Ass. Shell Structures symposium on non-linear behaviour of reinforced concrete spatial structures, Darmstadt, 1978, Wermer-Verlag, Dusseldorf, 1978, 89-100.

15. Cope R. J. et al. Finite element analysis of concrete bridge slabs. Proc. Int. Conf. Numer. Meth. Non-linear Problems, Swansea, 1980.

16. CooK, R. D. Concepts and applications of the finite element analysis. Wiley, New York, $1974,278,302$. 